\title{
TEM Based High Resolution and Low-Dose Scanning Electron Nanodiffraction Technique for Nanostructure Imaging and Analysis
}

\author{
Kyou-Hyun Kim ${ }^{1,2 * \dagger}$, Hui Xing ${ }^{1,2,3 *}$, Jian-Min Zuo ${ }^{1,2 \&}$, Peng Zhang ${ }^{4}$ and Haifeng Wang $^{4}$ \\ ${ }^{I}$ Department of Materials Science and Engineering, University of Illinois at Urbana-Champaign, Urbana, \\ Illinois 61801, USA \\ ${ }^{2}$ Frederick Seitz Materials Research Laboratory, University of Illinois at Urbana-Champaign, Urbana, Illinois \\ 61801, USA \\ ${ }^{3}$ School of Materials Science and Engineering, Shanghai Jiaotong University, Shanghai 200240, People's \\ Republic of China \\ ${ }^{4}$ Western Digital Technologies, Inc., 44200 Osgood Road, Fremont CA, 94539
}

\begin{abstract}
We report a high resolution and low-dose scanning electron nanodiffraction (SEND) technique for nanostructure analysis. The SEND patterns are recorded in a transmission electron microscope (TEM) using a low-brightness $\sim 2 \mathrm{~nm}$ electron beam with a $\mathrm{LaB}_{6}$ thermionic source obtained by a large demagnification of the condenser 1 lens. The diffraction pattern is directly recorded using a CCD camera optimized for low-dose imaging. A custom script was developed for calibration and automated data acquisition. The performance of low-dose SEND is evaluated using nanostructured Au as a test sample for the quality of diffraction patterns, sample stability and probe size. We demonstrate that our method provides an effective and robust way for recording diffraction patterns from nanometer-sized grains.
\end{abstract}

*The first two authors contributed equally to this work.

${ }^{\dagger}$ Current address: Advanced Process and Materials R\&BD Group, Korea Institute of Industrial Technology, Incheon 406-840, Republic of Korea

${ }^{\&}$ Corresponding author: Jian-Min Zuo, Professor (jianzuo@illinois.edu) 


\section{Introduction}

Many technologically important materials possess complex nanostructures and properties highly dependent on the structure. Transmission electron diffraction (TED) is an appropriate technique for complex nanostructure analysis because it is highly sensitive to local structure and it can be obtained using a small electron beam (Cowley, 1993; Spence and Zuo, 1992; Zuo et al., 2004; Zuo et al., 2003). Compared to the scanning electron microscope (SEM) based electron backscattering diffraction technique, the small interaction volume in TED allows for high spatial resolution. Furthermore, as TED patterns are formed mostly by elastically scattered electrons with sharp diffraction features, the diffraction pattern can be analyzed with greater accuracy.

Traditionally, TED is performed either by using parallel beam illumination with the help of a selected area aperture for selected area electron diffraction (SAED) or by using a focused beam for convergent beam electron diffraction (CBED). The electron nanodiffraction technique was pioneered by Cowley using a dedicated scanning transmission electron microscope (STEM) (Cowley, 2002; Cowley and Sundell, 1997). By using a small condenser aperture and optimized focus, Cowley demonstrated electron diffraction with a small convergent beam of $1 \mathrm{~nm}$ or smaller in diameter. In STEM, a sample image can be easily recorded by scanning the electron beam and detecting the scattered electrons using either a bright-field (BF) or an annular dark field (ADF) detector. Once the image is obtained, electron nanodiffraction patterns can be recorded by positioning the electron probe at selected sample areas. Because STEM imaging is performed in diffraction mode, no additional optical adjustment is needed between imaging and diffraction. Using this technique, Cowley demonstrated electron nanodiffraction for a number of interesting samples at resolution of $1 \mathrm{~nm}$ or less. In modern TEM instruments, a parallel electron beam of nanometers in diameter can be obtained with help of an additional condenser lens (minilens) (Zuo et al., 2004; Zuo et al., 2003). In a field emission gun (FEG) TEM, the small beam gives excellent coherent diffraction patterns. For complex nanostructure analysis, there is a need to record multiple diffraction patterns from desired sample areas. In this case, the switch from imaging mode when 
the probe is positioned to diffraction mode for diffraction pattern recording becomes a limiting factor. To overcome this issue, Kolb et al. obtained a nearly-parallel beam in STEM mode for coherent electron diffraction using a FEG TEM/STEM (Kolb et al., 2007). The reported probe sizes ranged between 15-50 $\mathrm{nm}$ at the specimen. Similarly, He and Nelson demonstrated nanoarea diffraction using a parallel beam of $80 \mathrm{~nm}$ in diameter obtained using Kohler illumination in STEM mode (He and Nelson, 2007). There are also efforts to combine nanobeam diffraction (NBD) performed in TEM with STEM operation. Alloyeau et al. (Alloyeau et al., 2008) and Ganesh et al. (Ganesh et al., 2010) have reported alignment methods where the probe is initially formed in NBD mode and kept after switching to STEM mode. The probe can be scanned to produce both BF and dark-field (DF) STEM images. The reported probe diameter ranges from 2 to $3 \mathrm{~nm}$. When this small probe is controlled by the Gatan ${ }^{\mathrm{TM}}$ software "STEM Diffraction Imaging", the system can be used to acquire diffraction patterns from an area on the sample on a pixel by pixel basis. Alternatively, Tao et al. reported a scanning electron nanodiffraction (SEND) technique in TEM mode by automating the diffraction pattern recording using a DigitalMicrograph (DM) (Gatan, CA) script and control of deflection coils in a JEOL (JEOLUSA, MA) 2010 FEG TEM (Tao et al., 2009). The reported electron probe has a diameter of about $1.7 \mathrm{~nm}$ and a convergence angle of 0.5 mrads obtained using a $4 \mu \mathrm{m}$ diameter condenser aperture.

All of above approaches for electron nanodiffraction rely on the FEG instruments. The beam current is on the order of tens of pico-ampere (pA), comparable to the beam current of a STEM probe. However, recording of electron diffraction patterns takes a longer time on the order of $\sim 0.1$ seconds compared to tens of $\mu$ s for STEM. Because of this, sample drift, heating, and damage are serious issues in the recording of SEND patterns (Malac et al., 2008).

Here, we report a low-dose method for SEND. Electron beams of $2 \sim 5 \mathrm{~nm}$ in full-width at halfmaximum (FWHM) and $0.1 \mathrm{pA}$ or less in beam current are obtained using a JEOL 2100 TEM with the $\mathrm{LaB}_{6}$ electron source. The small diameter beam is formed by using a strong condenser 1 lens setting. We demonstrate the performance of this probe for SEND using a nanostructured $\mathrm{Au}$ sample. In order to 
perform SEND, the microscope must be aligned, automated and calibrated for diffraction pattern recording. The procedures for doing this are also described here together with a discussion on the probe size.

\section{Electron probe formation}

A TEM equipped with a $\mathrm{LaB}_{6}$ thermionic emission gun, used for the work described here has illumination lens system consisting of three condenser lenses (CL), namely CL1, CL2 and condenser minilens $(\mathrm{CM})$, plus the objective lens pre-field (OL). The microscope is operated in the CBED mode with the CM lens excited, its excitation controlled by the alpha setting. Figure 1 shows the working of these lenses for forming a focused electron probe under the CBED mode. In this setup, the CL1 demagnifies the electron source image coming from the gun. The size of the electron beam crossover after the CL1 decreases as the excitation of the CL1 lens increases. The CL2 and CM lenses focus the electron beam onto the sample.

The probe diameter $d_{0}$ on the sample is determined by a combination of factors that include the source image diameter after CL1 $\left(d_{\mathrm{s}}\right)$. On the sample, the size of the source image is further demagnified $(\mathrm{M}<1)$ by the combination of CL2, CM and OL. The other factors in determining the probe size come from broadening by the effects of spherical aberration in the probe forming lenses $\left(d_{s a}\right)$ and condenser aperture diffraction $\left(d_{d}\right)$. The chromatic aberration $\left(d_{c}\right)$ of the probe forming lenses and the focusing error $\left(d_{f}\right)$ also contribute to the broadening of the probe diameter. The overall probe diameter can be expressed as the following (Spence and Zuo, 1992):

$$
\begin{aligned}
d_{0}^{2} & =d_{s}^{2} M^{2}+d_{d}^{2}+d_{s a}^{2}+d_{c}^{2}+d_{f}^{2} \\
& =d_{s}^{2} M^{2}+\left(\frac{0.6 \lambda}{\theta_{c}}\right)^{2}+\left(0.5 C_{s} \theta_{c}^{3}\right)^{2}+\left(\frac{\Delta E_{0}}{E_{0}} C_{c} \theta_{c}\right)^{2}+\left(2 \theta_{c} \Delta f\right)^{2}
\end{aligned}
$$


where $\lambda$ is the wave length of the electron beam, $\theta_{c}$ is the beam convergence angle, $C_{s}$ is the spherical aberration coefficient, $C_{c}$ is a chromatic aberration coefficient, and $\Delta f$ is defocus.

The size of the focused electron probe in TEM equipped with a $\mathrm{LaB}_{6}$ source is largely determined by the first term in equation 1, e.g., the electron source image size. The different probe sizes can be experimentally obtained by the different lens settings for CL1. For JEOL ${ }^{\circledR} 2100$ TEM used in this study, the focused beam diameter changes from $\sim 2$ to $\sim 40 \mathrm{~nm}$ when the spot size setting is changed from 5 to 1 for the CL1 lens. Apart from the source size contribution, $\Delta f=0$ when it is at focus, $\mathrm{M}$ is fixed by the alpha setting, and the contribution from $C_{c}$ is small. When a small condenser aperture is used, the diffraction broadening is larger than the contribution of spherical aberration.

Figure 2 shows an image of the electron probe formed using a $10 \mu \mathrm{m}$ condenser aperture in CBED mode with alpha $=1$. The intensity profile across the center of the probe gives a FWHM of $2.3 \mathrm{~nm}$.

\section{Microscope alignment}

Ideally, the center, i.e. the transmitted spot of (000) reflection in the diffraction pattern, should stay at the same location on the detector, while the electron probe is scanned across the sample. Improper alignment of microscope lenses, however, can lead to diffraction pattern shift during beam scanning, which affects the post diffraction data analysis. To minimize this effect, first, we follow the standard procedures for the microscope alignment and for correcting the shift-tilt purity of the illumination deflection coils. The alignment for shift-tilt purity separates beam shift from beam tilt in the beam deflection coil. The optical axis alignment of the objective lens is done through the use of bright tilt and high voltage centering. This is followed by adjustment of the intermediate lens focus, which can have a large effect on diffraction pattern shift.

Figure 3 illustrates the electron ray diagram for the direct beam in diffraction mode for (a) the strong, (b) the proper and (c) the weak intermediate lens settings. The ray paths labeled as '1', '2' and ' 3 ' respectively represent the electron beam coming from different positions during beam scanning. In 
Figures 3(a) and (c), the improper intermediate lens settings shift the position of the diffraction pattern for different incident beam positions on the sample. In contrast, the direct beam does not move under the proper setting for the intermediate lens, as shown in Figure 3(b).

The pattern movement can be minimized by changing the diffraction focus as follows. The procedures were developed for one TEM, but the principle applies to other TEMs.

(1) Align TEM following the standard alignment procedures for CL aperture, beam tilt, gun tilt, pivot point, etc.

(2) Find a vacuum area in TEM mode.

(3) Switch to diffraction mode. The direct beam will be seen on the screen. In this step, the direct beam is not necessarily sharp.

(4) Turn on the BEAM SHIFT to move the electron beam on the sample automatically without tilting the beam.

(5) Adjust the diffraction focus until the direct beam does not move.

In step 4, the electron beam can be manually shifted if the TEM has no automated beam-shift function. Once this alignment is completed, the diffraction focus is kept the same for subsequent SEND acquisition. In addition, the convergence angle of illumination beam does not cause pattern shift during the SEND acquisition.

\section{Scanning electron beam and scan calibration in TEM}

To automate SEND and diffraction pattern acquisition, we developed a script written in the DM script language. DM software also provides a control platform used to acquire, process and analyze images and spectrum data in TEM by running the DM scripts to customize tasks such as TEM operation and image manipulation. An electron microscope can be controlled using the scripts by communicating with the host processor built inside TEM with the correct microscope control plug-in installed. 
Scanning the electron beam in TEM mode is carried out by using the illumination deflection coils to control the beam shift through the script on the control computer. Two main commands are used for the scanning process: (1) EMGetBeamShift(Number $x$, Number y) and (2) EMSetBeamShift(Number $x$, Number y). The first command, EMGetBeamShift, retrieves the values of the illumination deflection coils and stores the values into the assigned real number $\mathrm{x}$ and $\mathrm{y}$. The second command, EMSetBeamShift, shifts the electron beam by the amounts of $\mathrm{x}$ and $\mathrm{y}$. The $\mathrm{x}$ and $\mathrm{y}$ values, however, only refer to the setting values of the electromagnetic lenses, which cannot be directly compared to real distances. Thus, the values need to be calibrated into distances in nanometers.

SEND requires two scanning vectors along the vertical and horizontal directions. Figure 4 shows the calibration procedures for establishing these two vectors. The calibration is carried out under a standard magnification in TEM mode. The reference value of $\left(\mathrm{x}_{1}, \mathrm{y}_{1}\right)$ is first obtained using EMGetBeamShift from the initial beam position, as shown in Figure 4. The electron beam is then horizontally shifted to '2', and $\left(\mathrm{x}_{2}, \mathrm{y}_{2}\right)$ are obtained. Using the calibrated magnification, the distance $(d)$ between ' 1 ' and ' 2 ' can be selected to a fixed value. Figure 4 shows an example for $d=400 \mathrm{~nm}$. From the above values, the horizontal scanning vector $\left(\mathbf{V}_{\mathbf{x}}\right)$ can be calculated as follows:

$$
\mathbf{V}_{\mathbf{x}}=\frac{1}{d}\left(x_{2}-x_{1}, y_{2}-y_{1}\right)(\mathrm{nm})
$$

Similarly, the vertical scanning vector $\left(\mathbf{V}_{\mathbf{y}}\right)$ can be calculated from $\left(\mathrm{x}_{1}, \mathrm{y}_{1}\right)$ and $\left(\mathrm{x}_{3}, \mathrm{y}_{3}\right)$ as follows:

$$
\mathbf{V}_{\mathbf{y}}=\frac{1}{d}\left(x_{3}-x_{1}, y_{3}-y_{1}\right)(\mathrm{nm})
$$

Once $\mathrm{V}_{\mathrm{x}}$ and $\mathrm{V}_{\mathrm{y}}$ are calibrated, the electron beam can be shifted to a specific position by the combination of two vectors using the command of EMSetBeamShift. 


\section{Automated scanning electron nanodiffraction}

The script we developed consists of two components; one for scan calibration, as shown in Sec. 4, and one for SEND data acquisition. For recording electron diffraction patterns, we used the Gatan ${ }^{\mathrm{TM}} \mathrm{CCD}$ camera designed for a $200 \mathrm{kV}$ electron source. The camera has a 4 megapixel ( $2 \mathrm{k} \mathrm{x} 2 \mathrm{k})$, Peltier-cooled, CCD chip. Its performance is optimized for biological applications through the use of an ultra-high sensitivity phosphor scintillator. The camera is mounted on-axis under the microscope column.

The developed SEND script uses the command of SSCGainNormalizedBinnedAcquire in order to record diffraction patterns during the scanning process. For our SEND experiment, we use 8 times for binning ( $256 \times 256$ pixels). The typical exposure time per diffraction pattern is 0.1 seconds. It takes about $\sim 5$ minutes to acquire $20 \times 20$ diffraction patterns.

\section{Scanning electron nanodiffraction and imaging of nanostructured Au}

We have applied our low-dose SEND technique to a nanostructured Au disk to test the performance, as shown in Figure 5(a). The scanning diffraction patterns were acquired over the area of $210 \times 210 \mathrm{~nm}^{2}$ in $30 \times 30$ pixels, corresponding to a step size of $7 \mathrm{~nm}$. The diffraction patterns were recorded over a period of 10 minutes. Figure 5(b) shows one of 900 diffraction patterns acquired from SEND. The diffracted beams appear as small disks corresponding to $4.2 \mathrm{mrad}$ in full convergence angle. As shown in the figure, the low-dose SEND enables recording of a diffraction pattern without using a beam stopper so that the full feature of the diffraction pattern can be recorded. To test the quality of SEND data and demonstrate the imaging capability of the developed technique, we integrated the diffraction intensity between two circles as marked in Figure 5(b) for the recorded diffraction patterns. The intensity sum for every single diffraction pattern is then mapped in the raster image, as shown in Figure 5(c). For the mapping, three regions of the diffraction pattern are selected as marked in Figures 5(c, d and e): (1) an annular area between the direct beam and the first ring (marked as 1), (2) the second ring (marked as 2), and (3) the 
remaining area of the third ring akin to the annular dark field (ADF) detector in STEM. For the first region (Figure 5(c)), the amorphous region (C film) has high intensity while the Au nanodisk appears in low intensity. This is expected since the amorphous scattering is strong where there are no Bragg spots from the Au nanodisk. Figure 5(d) shows the variation in the integrated intensity over the grains of Au nanoparticles. This reflects the orientation change across the grains. The ADF image also shows the intensity variations inside the Au nanodisk (Figure 5(e)). This can be explained by diffraction contrast coming from the low cutoff angle.

As shown in Figure 5, the geometry of the Au nanodisk is remarkably reproduced in the ADF images (Figure 5) compared to the bright-field image shown in Figure 5(a). This shows that both scan distortions and sample drift can be ignored. To check the sample drift during SEND, we recorded both the initial specimen image and the final specimen image after the data acquisitions, as shown in Figure 6. We calculated the sample drift from two images, and the result shows that the drift is $3 \mathrm{~nm}$ or less during a period of 10 minutes in both horizontal and vertical directions. No sample damage or contamination was observed due to the low current beam used here.

Further image processing can be applied in order to extract structural information from the set of scanning diffraction patterns. For example, we calculated the cross-correlation coefficient for all patterns and grouped the patterns according to their correlation. Once the diffraction patterns are separated into groups, the average diffraction pattern can be calculated from these groups to determine the sample regions more precisely. Average diffraction pattern can be also directly obtained with the help of imaging such as the ones shown in Figure 5. The ADF image of the Au nanodisk obviously shows seven different regions. For these regions, the diffraction patterns are extracted, averaged and shown in Figure 7. Because the quality of averaged diffraction patterns is much more enhanced than a single diffraction pattern, the averaged pattern can significantly improve the reliability of diffraction pattern indexing. Further work is underway to develop an automated diffraction indexing procedure for low-dose SEND patterns. 


\section{Conclusions}

We have developed a high resolution, low dose, scanning electron nanodiffraction method in TEM. This is achieved by establishing a scan calibration and automated diffraction pattern acquisition procedure. The electron probes of as small as $2 \mathrm{~nm}$ are formed using a $\mathrm{LaB}_{6}$ thermionic emission source, where the beam current can be reduced continuously by adjusting the gun heating current. For the $2 \mathrm{~nm}$ probe, we demonstrated SEND from a nanostructured Au disk. The quality of diffraction patterns is demonstrated by imaging using the SEND data and the quality of spatially averaged diffraction patterns. In the last case, we show that SEND enables a new way for obtaining selected area electron diffraction without the use of an aperture.

\section{Acknowledgements}

The work is supported by a grant from Western Digital Technologies, Inc. and DOE BES (Grant No. DEFG02-01ER45923). In addition, Hui Xing is supported by the China Scholarship Council (CSC) and the National Natural Science Foundation of China (Grant No. 51101099). Electron microscopy was carried out at the Center for Microanalysis of Materials at the Frederick Seitz Materials Research Laboratory of the University of Illinois at Urbana-Champaign.

\section{Figure Captions:}

Figure 1. Ray diagram of illumination system for CBD mode in the LaB6 TEM used in this study. Figure 2. Electron probe formed using a $10 \mu \mathrm{m}$ condenser aperture in CBD mode.

Figure 3. Effect of intermediate lens setting on the movement of diffraction pattern during beam scanning. Figure 4. Schematic of calibration procedures for SEND.

Figure 5. (a) A BF image of nanostructured Au disk and (b) a selected diffraction pattern acquired from SEND. The diffraction intensity is integrated for the areas of 1,2, and 3 represented in (b). The corresponding maps are shown in (c), (d) and (e), respectively. 
Figure 6. Sample drift during SEND. (a) is the initial position, and (b) is the final position after SEND.

Figure 7. Representative diffraction patterns for the seven marked sample regions obtained from the recorded SEND patterns.

\section{References}

Alloyeau, D., Ricolleau, C., Oikawa, T., Langlois, C., Le Bouar, Y., Loiseau, A., 2008. STEM nanodiffraction technique for structural analysis of CoPt nanoparticles. Ultramicroscopy 108, 656-662.

Cowley, J.M., 1993. Electron Diffraction Techniques. International Union of Crystallography.

Cowley, J.M., 2002. Electron nanodiffraction methods for measuring medium-range order. Ultramicroscopy 90, 197-206.

Cowley, J.M., Sundell, F.A., 1997. Nanodiffraction and dark-field STEM characterization of singlewalled carbon nanotube ropes. Ultramicroscopy $68,1-12$.

Ganesh, K., Rajasekhara, S., Bultreys, D., Zhou, J.-P., Ferreira, P., 2010. Automated Local Texture and Stress Analysis in $\mathrm{Cu}$ Interconnects Using D-STEM and Precession Microscopy. Microscopy and Microanalysis 16, 1728-1729.

He, H., Nelson, C., 2007. A method of combining STEM image with parallel beam diffraction and electron-optical conditions for diffractive imaging. Ultramicroscopy 107, 340-344.

Kolb, U., Gorelik, T., Kübel, C., Otten, M.T., Hubert, D., 2007. Towards automated diffraction tomography: Part I—Data acquisition. Ultramicroscopy 107, 507-513.

Malac, M., Beleggia, M., Taniguchi, Y., Egerton, R.F., Zhu, Y., 2008. Low-dose performance of parallelbeam nanodiffraction. Ultramicroscopy 109, 14-21.

Spence, J.C.H., Zuo, J.M., 1992. Electron microdiffraction. Plenum Press.

Tao, J., Niebieskikwiat, D., Varela, M., Luo, W., Schofield, M.A., Zhu, Y., Salamon, M.B., Zuo, J.M., Pantelides, S.T., Pennycook, S.J., 2009. Direct imaging of nanoscale phase separation in $\mathrm{La}_{0.55} \mathrm{Ca}_{0.45} \mathrm{MnO}_{3}$ : relationship to colossal magnetoresistance. Physical Review Letters 103. 
Zuo, J.M., Gao, M., Tao, J., Li, B.Q., Twesten, R., Petrov, I., 2004. Coherent nano-area electron diffraction. Microscopy Research and Technique 64, 347-355.

Zuo, J.M., Vartanyants, I., Gao, M., Zhang, R., Nagahara, L.A., 2003. Atomic Resolution Imaging of a Carbon Nanotube from Diffraction Intensities. Science 300, 1419-1421. 
Figures

Figure 1

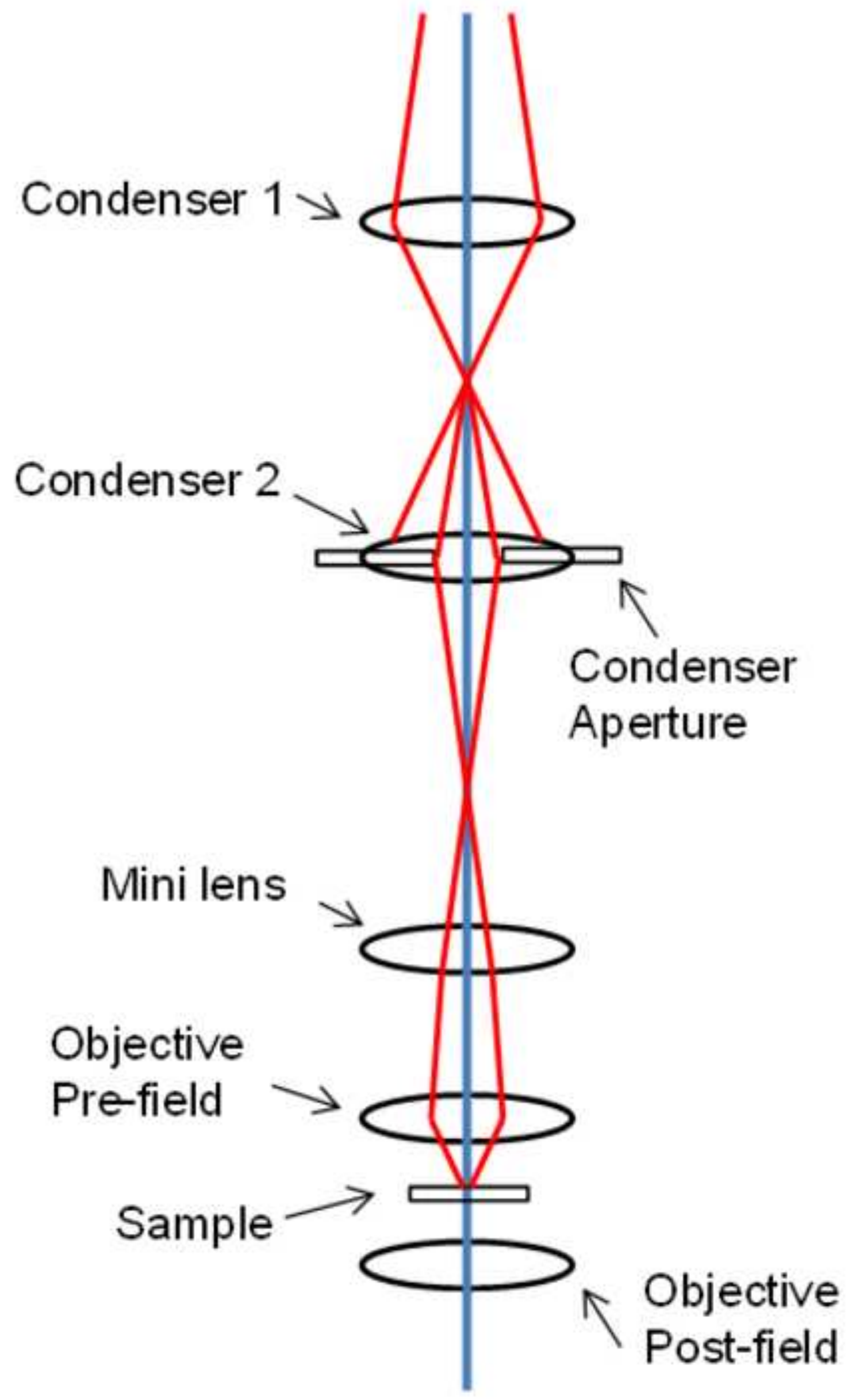




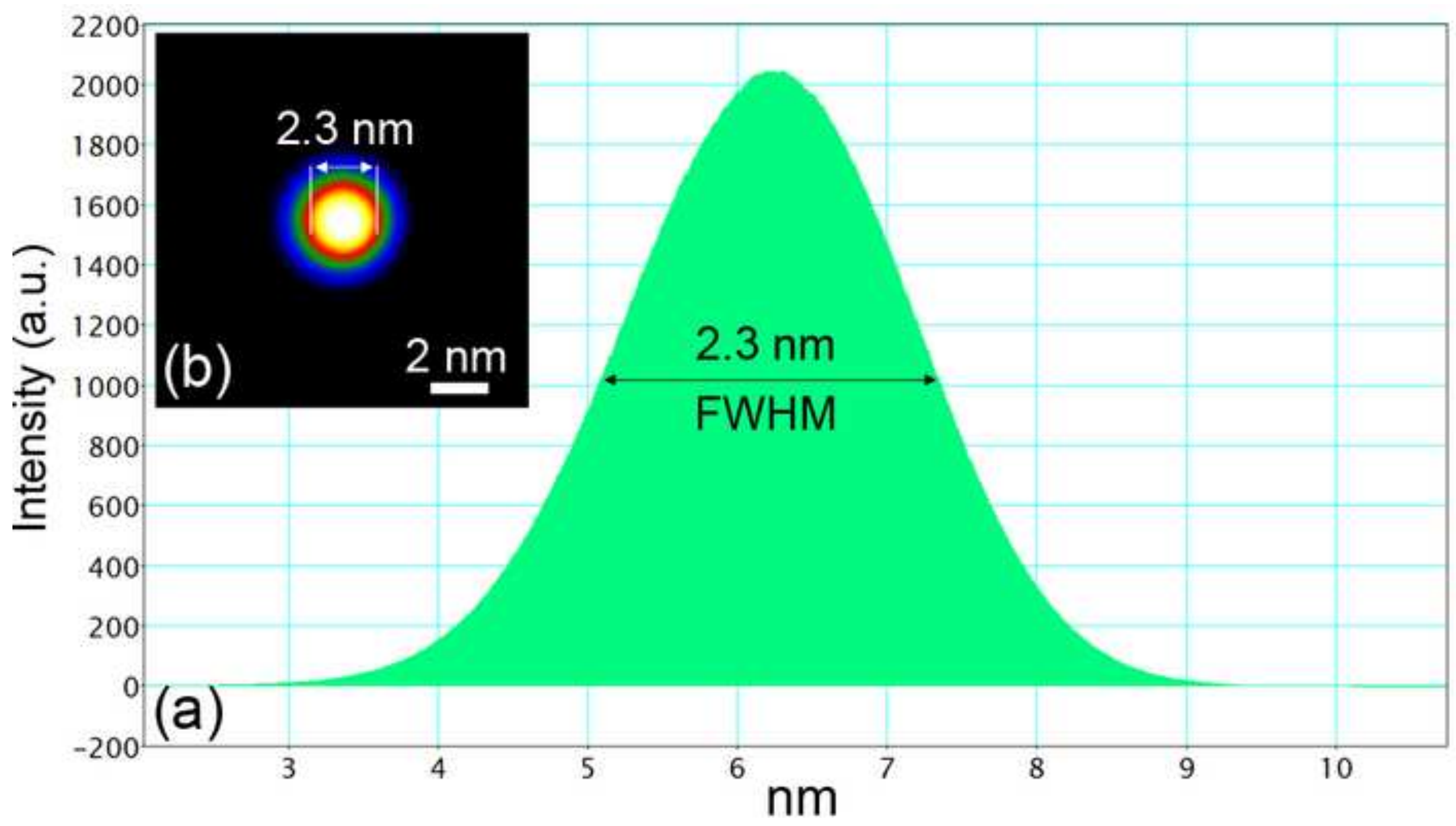




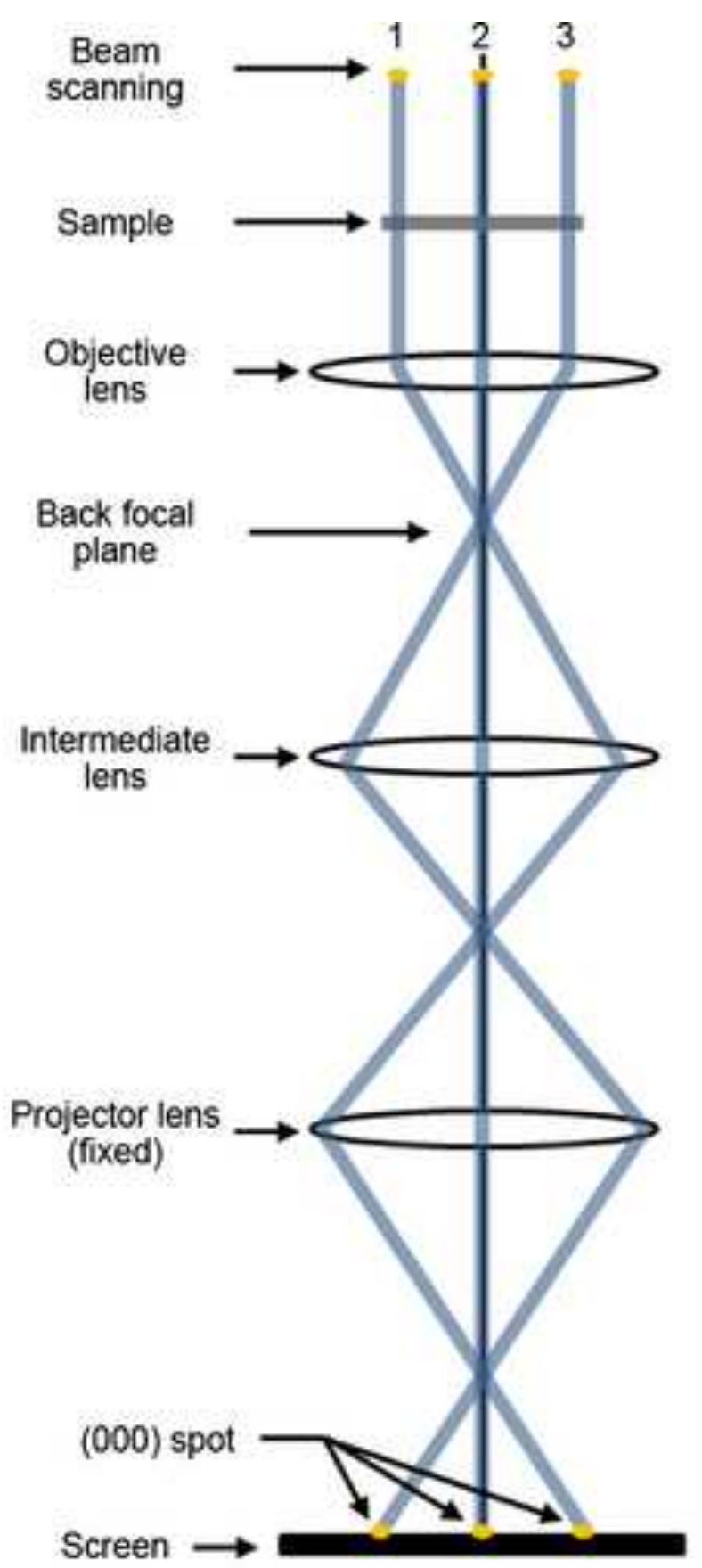

(a) strong intermediate lens setting

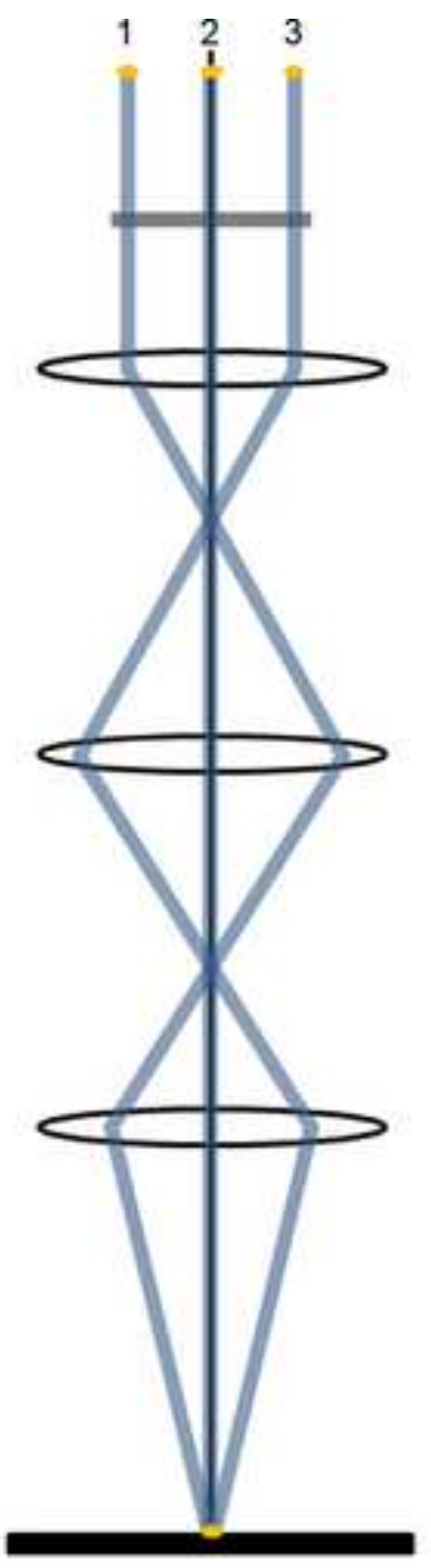

(b) proper intermediate lens setting

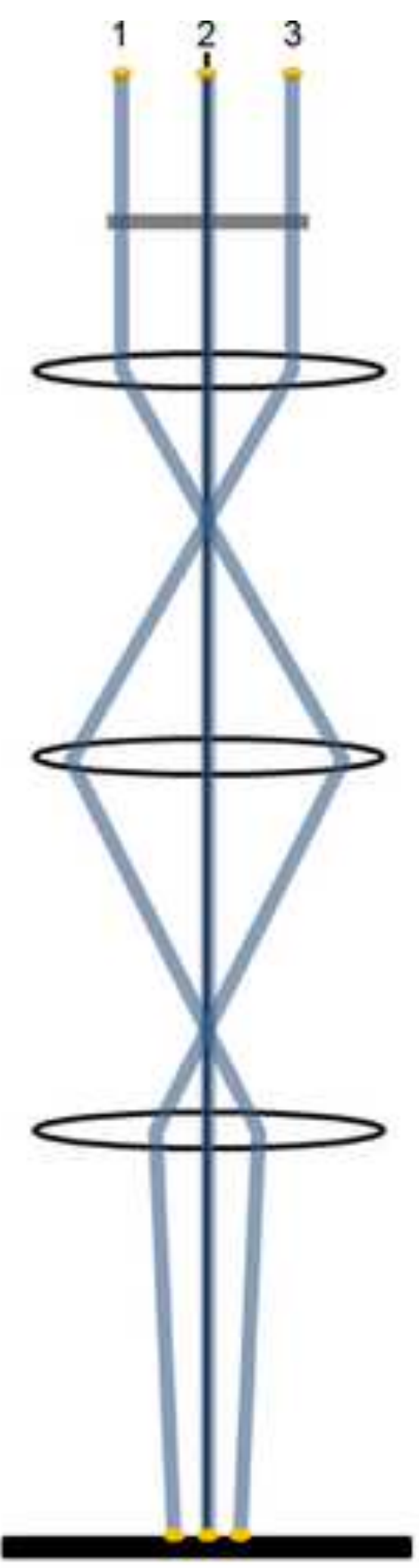

(c) strong intermediate lens setting 


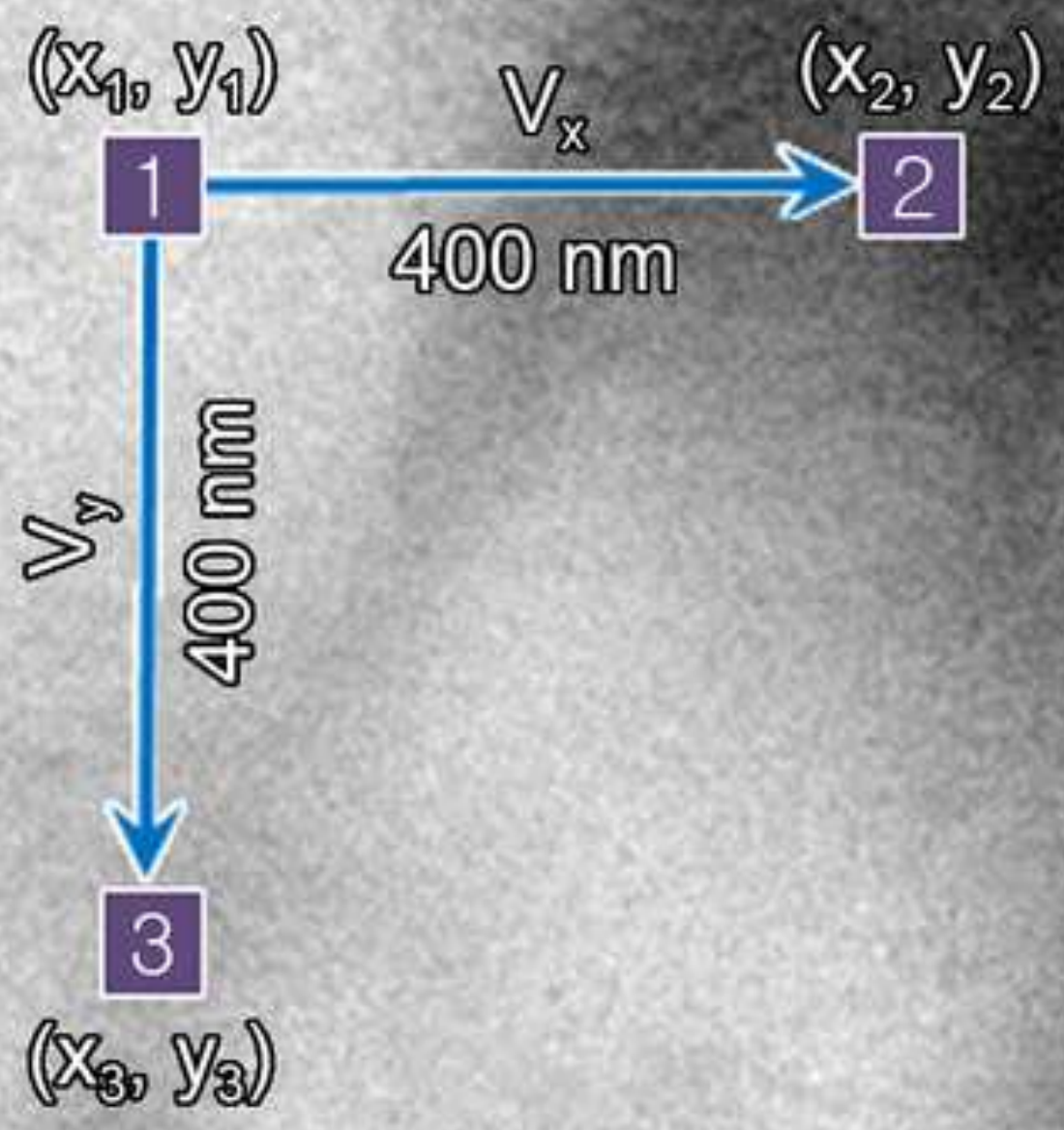

\section{0 nm}


Figure 5
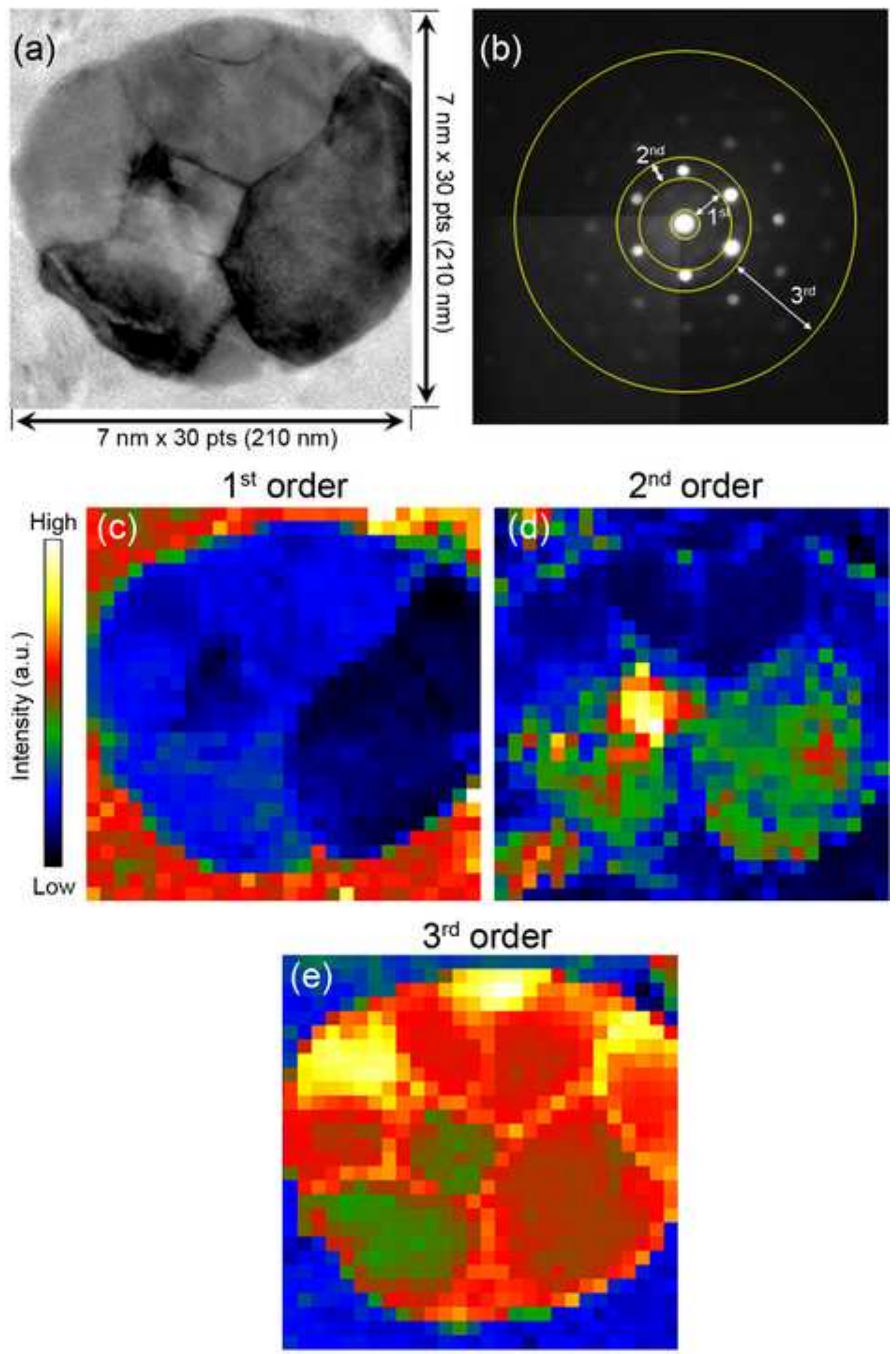


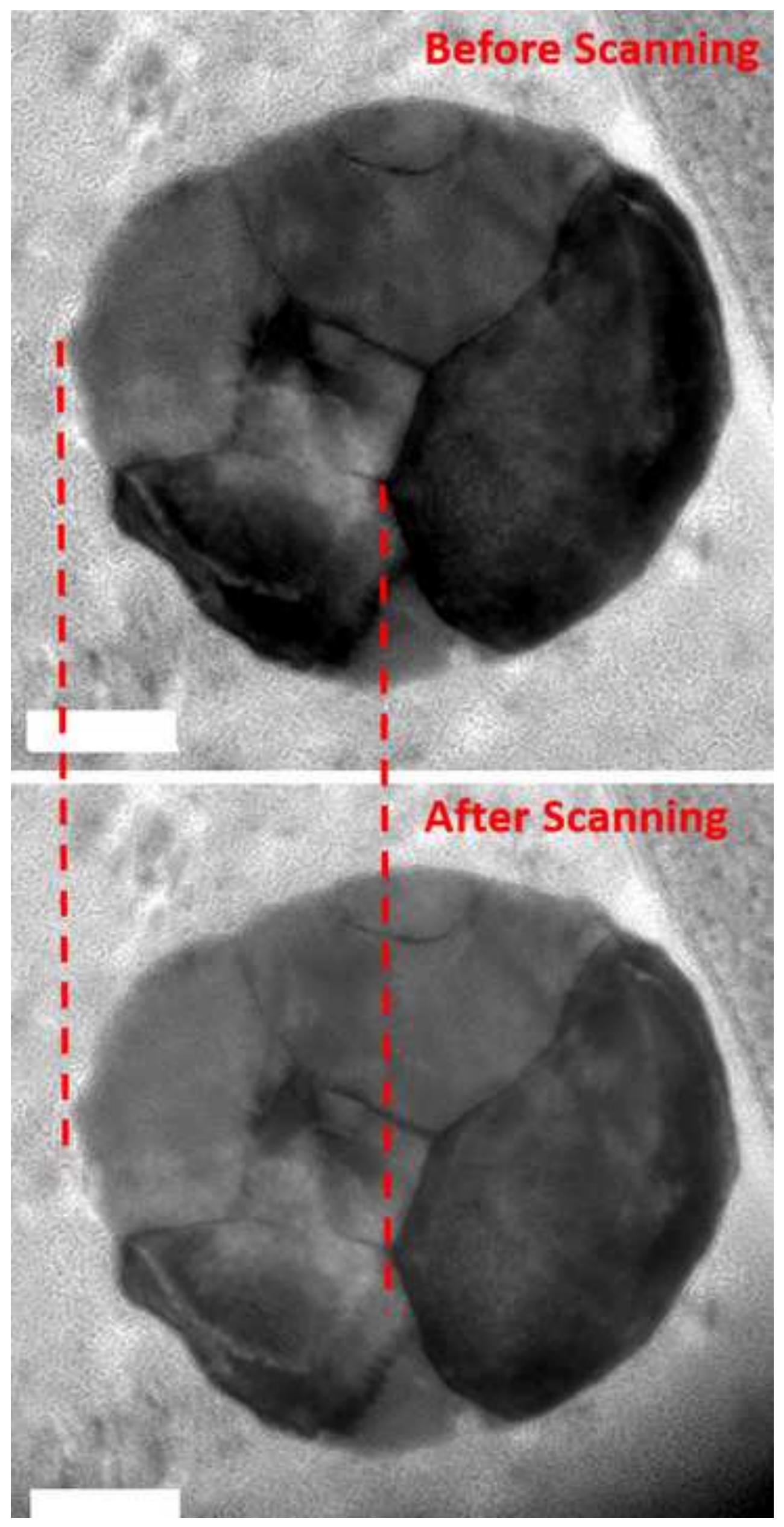

\section{Figure 6}




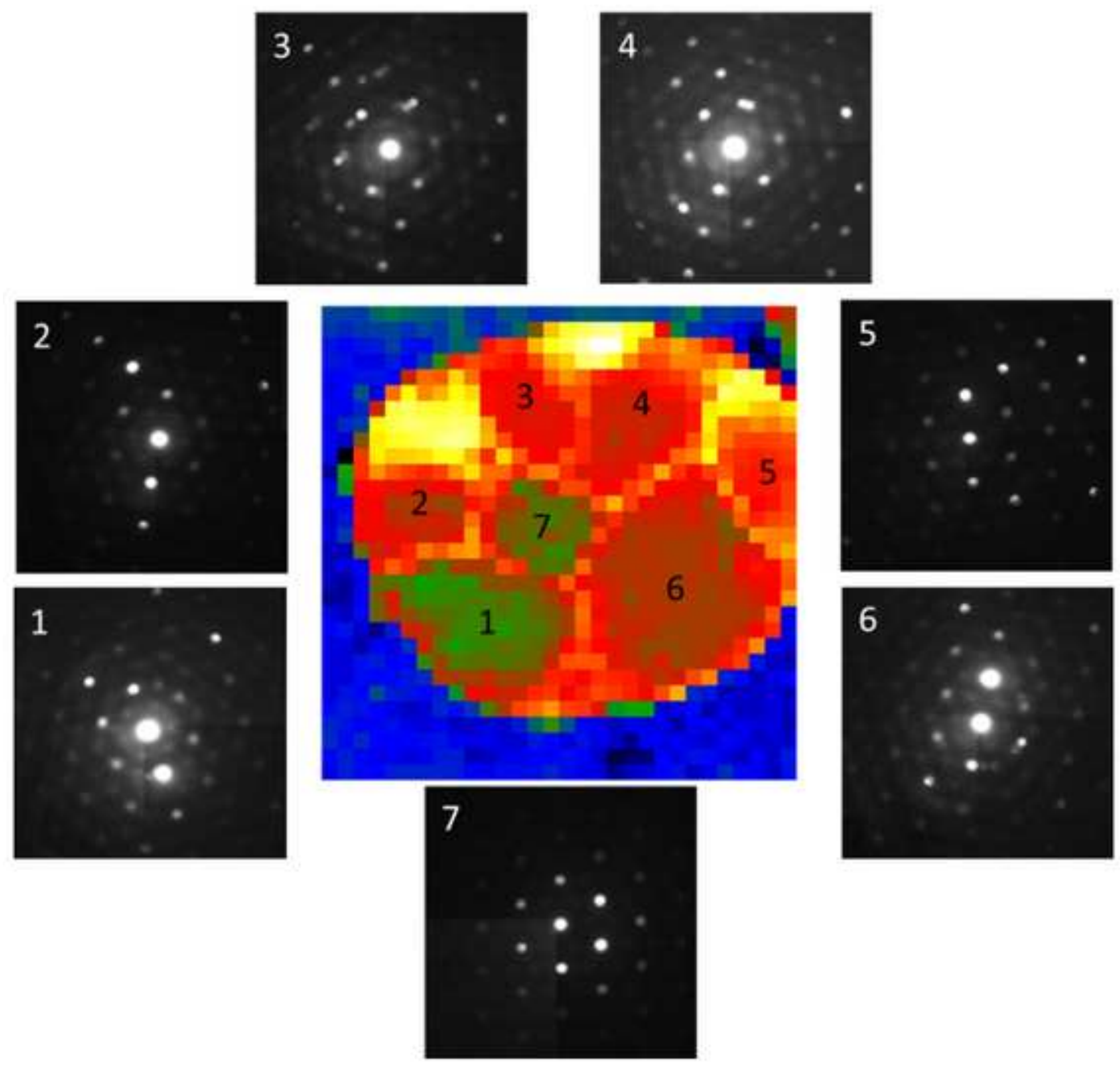

$$
\text { . }
$$

